\title{
Formation of Planetesimals by Burgers Vortex
}

\author{
Martin G. Abrahamyan \\ Department of Physics, Yerevan State University, Yerevan, Armenia
}

Email address:

martin.abrahamyan@ysu.am

\section{To cite this article:}

Martin G. Abrahamyan. Formation of Planetesimals by Burgers Vortex. International Journal of Astrophysics and Space Science. Vol. 4, No. 3, 2016, pp. 26-31. doi: 10.11648/j.ijass.20160403.11

Received: August 23, 2016; Accepted: September 1, 2016; Published: September 21, 2016

\begin{abstract}
The effect of a Burgers vortex on formation of planetesimals in a protoplanetary disc in local approach is considered. It is shown that there is not any circular orbit for rigid particles in centrifugal balance; only stable position in Burgers vortex under the influence of centrifugal, Coriolis, pressure gradient and Stokes drag forces is the center of vortex. The two-dimensional anticyclonic Burgers vortex with homogeneously rotating kernel and a converging radial stream of substance can effectively accumulate in its nuclear area the meter-sized rigid particles of total mass $\sim 10^{28} \mathrm{~g}$ for characteristic time $\sim 10^{6} \mathrm{yr}$.
\end{abstract}

Keywords: Protoplanetary Disc, Burgers Vortex, Planetesimals

\section{Introduction}

The radial dependence of infra-red, sub $\mathrm{mm}$ and $\mathrm{cm}$ radiation of protoplanetary disks analyses show that vortices serve as incubators for growth dust particles and formation of planetesimals [1]. Formation of planetesimals from micron size dust grains possibly, involves many physical processes [2]. It is accepted that growth of particles in dusty circumstellar discs is hierarchical [3, 4]. The initial stage of growth probably proceeds through the nucleation of sub-micron-sized dust grains from the primordial nebula, which then forms the monomers of fractal dust aggregates up to $\sim 1 \mathrm{~mm}$ to $\sim 10 \mathrm{~cm}$ for characteristic time of an order of $10^{3}$ years. The further growth of particles by this mechanism is stopped by collisional destruction process $[5,6]$. In this stage the dynamics of particles and coagulation are regulated by van der Waals forces and the Brownian motion. The best astrophysical evidence for grain growth to specified sizes is the detection of $3.5 \mathrm{~cm}$ dust emission from the face-on disk of radius $225 \mathrm{AU}$ round classical T Tauri star TW Hya (age $\sim 5-10$ Myr), located 56 pc away [7]. When the planetesimals reached a size of about $1 \mathrm{~km}$, they began to attract other smaller bodies due to their gravity.

In standard models of protoplanetary disks, gas pressure decreases along radius. Gas in a disk practically moves on sub-Keplerian speeds. Rigid particles, under the action of a head wind drags, lose the angular momentum and energy. As a result, the $\sim 10 \mathrm{~cm}$ to meter-sized particles drift to the central star for hundreds of years, that is, much less than the lifetime of a disk which makes several millions of years [8,9].

Long-lived vortical structures in gas disk are a possible way to concentrate the $\sim 10 \mathrm{~cm}$ to meter sized particles and to grow up them in planetesimal. Similar effect of vortices on the Earth observed in special laboratories, and also in the ocean. For example, the oceanic vortices trap larval fish off the coast of Western Australia [10].

In some areas of the stratified protoplanetary disks the current has 2D-turbulent character. An attractive feature of such hydrodynamic current consists in the fact that in it, through a background of small whirlpools, long-living vortices will spontaneously be formed without requirement of special initial conditions [11-13]. In laboratory experiments [14], formation of Burgers vortex which will be considered in the present work is often observed in 2D-turbulent flows. If protoplanetary disks are capable of producing 2D-turbulent flow, they can form long-living large-scale vortices which in due course lives up to an order of hundred orbital periods. Anticyclonic vortices in a protoplanetary disk merge with each other and amplify, while cyclonic ones are destroyed by a shear flow.

In an anticyclone, rigid particles are grasped by force of Coriolis directed to the center of a vortex. If the vortex survives $\sim 100$ rotations in a nebulae with solar mass, the quantity of the grasped particles can reach masses of planets (several masses of the Earth). Existence of long-living vortices in the protoplanetary disk, drifting of external regions of a disk, allows accumulation of the mass necessary for formation of a kernel of a giant planet $[15,16]$. 


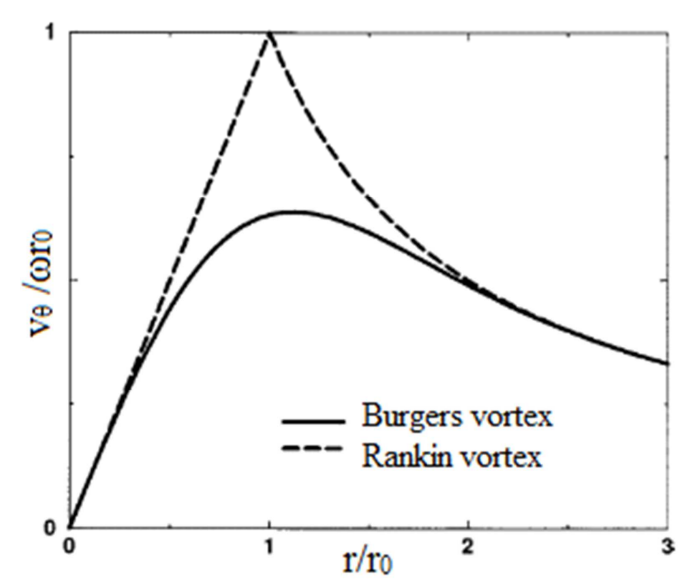

Fig. 1. Rotational velocity profiles of Burgers and Rankin vortices.

In the present work the Burgers vortex in a protoplanetary disk and its role in a problem of formation of planetesimals will be considered.

The vortex of Burgers in cylindrical system of co-ordinates $(\mathrm{r}, \theta, \mathrm{z})$ is defined as

$$
\mathrm{v}_{\mathrm{r}}=-\mathrm{Ar}, \mathrm{v}_{\theta}=\omega \mathrm{r}_{0}^{2}\left[1-\exp \left(-\mathrm{r}^{2} / \mathrm{r}_{0}^{2}\right)\right] / \mathrm{r}, \mathrm{v}_{\mathrm{z}}=2 \mathrm{Az}
$$

This represents a vortex with a converging stream of substance to its center where A characterizes a converging stream, and $\omega$ and $r_{0}$ - circulation and the size of a trunk of a vortex. Rotation in the field of a vortex trunk is almost rigid-state and on the big distances a profile of rotational speed falls down under the hyperbolic law (fig. 1). The asymptotic behavior of Burgers vortex in small and big distances from the vortex center represents the Rankin vortex [17, 18]. The maximum rotational speed in Burgers vortex is equal $0.638 \omega \mathrm{r}_{0}$ which is reached at $r / r_{0}=1.121$. At distance $r_{\text {eff }}=4.5 r_{0}$, rotational speed makes one-third of the maximum value. Conditionally we will name this distance "effective radius" of Burgers vortex.

\section{The Magnitude of Some Parameters of a Disk}

We consider the Burgers vortex in viscous axially-symmetrical accretion disk with effective temperature $\mathrm{T}$, gas volume density $\rho$, of almost Keplerian rotation. The sound speed in gas is estimated by the formula

$$
\mathrm{c}_{\mathrm{s}}=\sqrt{ } \gamma \mathrm{kT} / \mathrm{m}_{\mathrm{H}} \approx(\gamma \mathrm{T} / 100 \mathrm{~K})^{1 / 2} \mathrm{~km} / \mathrm{s},
$$

where $\gamma=1.4$ - the gas adiabatic index, $\mathrm{k}$ - Boltzmann constant, $\mathrm{m}_{\mathrm{H}}$ - hydrogen atom mass.

In a vertical direction, the gas is in hydrostatic balance with a characteristic scale height

$$
H \sim \frac{c_{s}}{\Omega} \approx 0.03\left(\frac{T}{100 K}\right)^{1 / 2}\left(\frac{M_{\odot}}{M}\right)^{1 / 2}\left(\frac{R}{A U}\right)^{3 / 2} A U .
$$

The superficial density of the gas in a disk can be estimated as $\Sigma \approx 2 \mathrm{H} \rho$.
In $\alpha$ - model of accretion disk [19], the expense of gas occurs with a speed $\mathrm{dm} / \mathrm{dt}=3 \pi v \Sigma$, where $v$ - kinematic viscosity of gas $-v=\alpha c_{s} H$.

The characteristic dynamic time scale of a disk is:

$$
\tau \sim \frac{1}{\Omega} \approx \frac{1}{5}\left(\frac{M_{\odot}}{M}\right)^{1 / 2}\left(\frac{R}{A U}\right)^{3 / 2} y r .
$$

For Keplerian disk, radial momentum equation solution yields to a difference between the speeds of rigid particles and surrounding gas [20]. In a thin gas disk $\left(\mathrm{c}_{\mathrm{s}} \ll \Omega R\right)$, rigid particles drift relative to gas with a speed

$$
\frac{\Delta v}{c_{s}} \sim \frac{c_{s}}{\Omega R} \approx 0.03\left(\frac{T}{100 K}\right)^{1 / 2}\left(\frac{M_{\odot}}{M}\right)^{1 / 2}\left(\frac{R}{A U}\right)^{1 / 2} .
$$

At $c_{\mathrm{s}} \sim 1 \mathrm{~km} / \mathrm{s}$, typical drift speed is of order $30 \mathrm{~m} / \mathrm{s}$. The characteristic scale of drift time [8,9] almost by two orders surpasses dynamic time $\tau$

$$
\tau_{\mathrm{d}} \sim \mathrm{r} / \Delta \mathrm{v} \sim(\text { R/A.E. }) 10^{2} \mathrm{yr} \text {. }
$$
[21]

Dust settled on a midplane of a disk for a characteristic time

$$
\tau_{\mathrm{s}} \sim \Sigma / \alpha \Omega \rho^{*},
$$

where $\rho^{*}$ is the mass density of a particle, and characteristic time between collisions of rigid particles among themselves is estimated as

$$
\tau_{\mathrm{col}} \sim \mathrm{D} \rho * / \Sigma * \Omega,
$$

where $\mathrm{D}$ is the diameter of a particle, $\Sigma^{*}$ is the superficial density of rigid particles in a disk which is more than by two orders less than a disk $\Sigma$. For metre-size rigid particles, this time is an order $\sim 5 y$ r. Therefore for creation of rigid particles with the sizes significantly surpassing the meter size, it is necessary to provide an environment with high concentration of particles in rather small regions. Such environment is provided by vortices.

Particles in a vortex are exposed to actions of centrifugal, Coriolis, drag forces, and in a smaller measure, to gradient forces of pressure. If centrifugal force deletes a particle from the vortex centre, drag and Coriolis forces in an anticyclonic Burgers vortex are directed to its centre. Acceleration of a rigid particle is directed to the vortex centre if the vortex angular speed $\omega=|\mathrm{d} \theta / \mathrm{dt}|$ at a small friction is less than $2 \Omega$,

$$
\omega<2 \Omega
$$

- a condition which is always carried out in practice.

Viscous dissipation and orbital shear limit the sizes of a vortex. Viscous dissipation destroys vortices of sizes less than viscous scale [16]

$$
L_{v}=\frac{\alpha c_{s} H}{v_{\theta}} \approx 0.003\left(\frac{\alpha}{0.01}\right)\left(\frac{0.1 c_{s}}{v_{\theta}}\right)\left(\frac{M_{\odot}}{M}\right)^{1 / 2}\left(\frac{R}{A U}\right)^{3 / 2} A U,
$$

where $\mathrm{v}_{\theta}$ - rotational speed of a vortex. 
The Keplerian shear flow forbids formation of circular structures with the sizes larger than shear length scale [22],

$$
\mathrm{L}_{\text {shear }}=\sqrt{v_{\theta}\left|\frac{d \Omega}{d R}\right|^{-1}} \approx 0.05\left(\frac{v_{\theta}}{0.1 c_{s}}\right)^{1 / 2}\left(\frac{M_{\odot}}{M}\right)^{1 / 4}\left(\frac{R}{A U}\right)^{5 / 4} A U .
$$

The vortices, whose sizes surpass $\mathrm{L}_{\text {shear, }}$ are extended in an azimuthal direction that allows them to survive longer. In [23] we have shown the possibility of formation in a disk extended in an azimuthal direction three-axis ellipsoidal vortex, with a linear field of circulation, similar to Riemann S ellipsoids [24]. However, note that in a disk round the central star of solar mass, at distance 30AU, the vortex of characteristic speed of rotation $0.01 \mathrm{c}_{\mathrm{s}}$, can be circular and have the size of an order $1 \mathrm{AU}$.

In a gas disk, drag force on rigid particles from gas is exposed, which, depending on size of a particle, is expressed either by Stokes, or Epstein's formula (see, for example, [15]). If the sizes D particles are small in comparison with gas mean free path, such particles are exposed to the Epstein drag force:

$$
F=\frac{\rho c_{s}}{\rho^{*} D} \Sigma *(\boldsymbol{v}-\boldsymbol{u})
$$

where $v$ - velocity of gas, $u$ - velocity of rigid particle. Particles of the big sizes are exposed to the Stokes drag force (see (15)).

\section{The Burgers Vortex in Local Frame of Reference}

Let's use local approach, choosing frame of reference, rotating with a disk with angular speed $\Omega_{0}$ at distance $R_{0}$ round the central star of mass M. In this approach, assuming the sizes of a vortex are much smaller than the distance $R_{0}$, we will choose Cartesian system of co-ordinates with center O (Fig. 2), directing the $\mathrm{Y}$ axis to a star, and the $\mathrm{X}$ axis in direction of Keplerian flow of gas. We will present the disk rotation as $\Omega(R)$ $\propto R{ }^{q}$. In case when only the gravitation of the central star operates, rotation will be Keplerian with $q=3 / 2$, and for homogeneously rotating disk $q=2$, i.e. $2 \geq q \geq 3 / 2$.

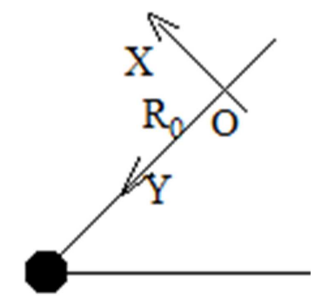

Fig. 2. Local frame of reference.

In the chosen system of reference, the substance stream has $\mathrm{X}$ component of speed $-\mathrm{iq} \Omega_{0} \mathrm{y}$, centrifugal force is compensated by radial component of gravitation of the central star at distance $R_{0}$, in other points their sum gives the tidal force $\mathrm{j} 3 \Omega_{0}{ }^{2} \mathrm{y}$. The vertical component of gravitation $-\Omega_{0}{ }^{2} z$ is returning force along axis $Z$.
At first we will consider a gas disk round the central body. In local approach, the equation stationary isentropic shear flow of gas taking into account viscosity will be described by Navier-Stokes and continuity equations

$$
\begin{gathered}
(\mathrm{v} \nabla) \mathrm{v}=\mathrm{j} 3 \Omega_{0}{ }^{2} \mathrm{y}-\mathrm{k} \Omega_{0}{ }^{2} \mathrm{z}-2 \Omega_{0} \times \mathrm{v}-\nabla \mathrm{h}+\mathrm{v} \Delta \mathrm{v} \\
\nabla(\rho \mathrm{v})=0,
\end{gathered}
$$

where $\mathrm{h}$ - specific enthalpy $\left(\mathrm{h}=\int \rho^{-1} \mathrm{dp}\right), \mathrm{i}, \mathrm{j}$ and $\mathrm{k}$ - Cartesian orts. The first term in the right side of equation (12) is, as noted above, tidal acceleration in a plane of the disk, the second term is vertical component of gravitation, the third is acceleration of Coriolis, the last is a viscous stress.

In the chosen Cartesian co-ordinate system the Burgers vortex will be presented in the form

$$
\begin{gathered}
\mathrm{v}_{\mathrm{x}}=-\mathrm{Ax}-\omega \mathrm{r}_{0}{ }^{2} \mathrm{y}\left[1-\exp \left(-\mathrm{r}^{2} / \mathrm{r}_{0}{ }^{2}\right)\right] / \mathrm{r}^{2}, \\
\mathrm{v}_{\mathrm{y}}=-\mathrm{Ay}+\omega \mathrm{r}_{0}{ }^{2} \mathrm{x}\left[1-\exp \left(-\mathrm{r}^{2} / \mathrm{r}_{0}{ }^{2}\right)\right] / \mathrm{r}^{2}, \\
\mathrm{v}_{\mathrm{z}}=2 \mathrm{Az},
\end{gathered}
$$

where $r^{2} \equiv x^{2}+y^{2}$.

It is easy to check that solutions (14) satisfy to the continuity equation at $\mathrm{d} \rho / \mathrm{dt}=0$ - that is comprehensible in local approach.

Substituting (14) in the equation (12), we will receive expression for specific enthalpy $\mathrm{h}(\mathrm{x}, \mathrm{y}, \mathrm{z})$. However we will not present the bulky expression for $\mathrm{h}(\mathrm{x}, \mathrm{y}, \mathrm{z})$. We will only describe spatial profiles of gradient forces $\partial \mathrm{h} / \partial \mathrm{x}$ and $\partial \mathrm{h} / \partial \mathrm{y}$ in $\mathrm{X}, \mathrm{Y}$ plane in anticyclonic Burgers vortex (fig. 3).
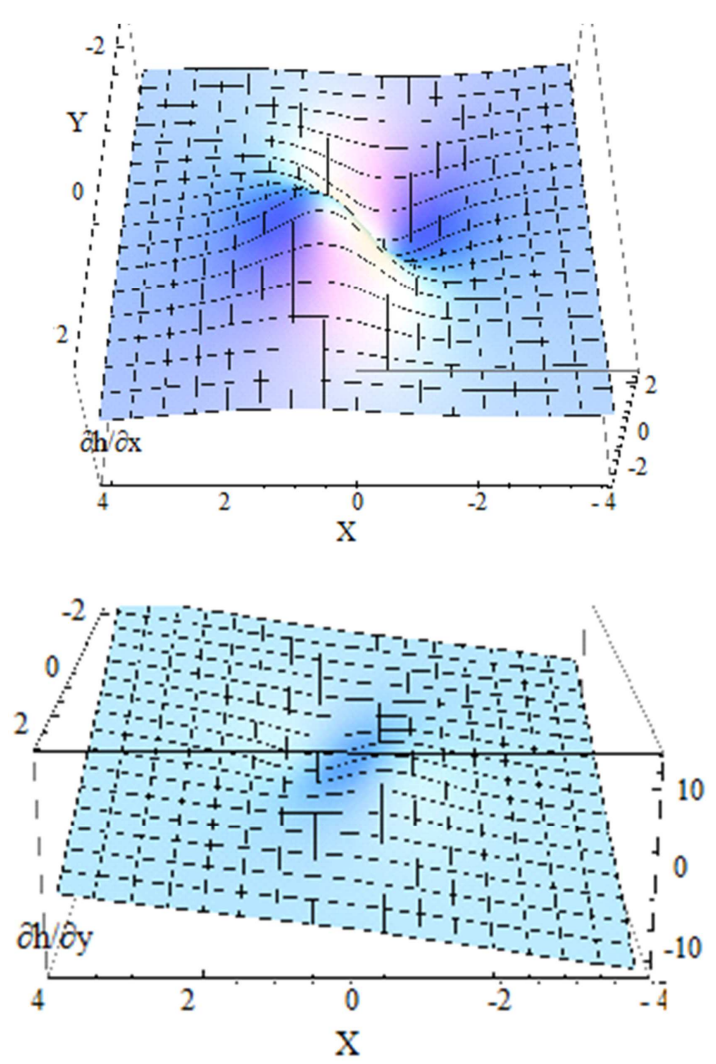

Fig. 3. Profile of pressure gradient force $T h$ which in an anticyclonic Burgers vortex forces to rotate disc matter clockwise. 


\section{The Dynamics of Rigid Particles in Burgers Vortex}

In the present work, we will be limited to studying two-dimensional dynamics of dust rigid particles in a Burgers vortex taking into account action pressure gradient force $\nabla$ h, tidal force, forces of Coriolis and a friction. Thus we will neglect the influence of rigid particles on dynamics of gas, and also the interaction of rigid particles among themselves.

Let's consider that the sizes of particles D considerably surpass the mean free path of molecules of gas, therefore we will describe the friction of rigid particles with gas by Stokes drag force

$$
\boldsymbol{f}=\beta(\mathbf{v}-\mathbf{u})
$$

where

$$
\beta \equiv 18 \rho v / \rho^{*} \mathrm{D}^{2},
$$

$\mathbf{u}$ - velocity of a particle:

$$
\mathbf{u}=(\mathrm{dX} / \mathrm{dt}, \mathrm{dY} / \mathrm{dt})
$$

$\mathrm{X}, \mathrm{Y}$ - particle co-ordinates.

The equation of motion of dust particles in the accepted approach looks like:

$$
\begin{gathered}
\mathrm{du} \mathrm{u}_{\mathrm{X}} / \mathrm{dt}=2 \Omega_{0} \mathrm{u}_{\mathrm{y}}-\beta\left(\left.\mathbf{v}_{\mathrm{x}}\right|_{\mathbf{r}=(\mathrm{X}, \mathrm{Y})}-\mathrm{u}_{\mathrm{x}}\right)-\partial \mathrm{h} /\left.\partial \mathrm{x}\right|_{\mathbf{r}=(\mathrm{X}, \mathrm{Y}),}( \\
\mathrm{du} \mathrm{y}_{\mathrm{y}} / \mathrm{dt}=3 \Omega_{0}^{2} \mathrm{Y}-2 \Omega_{0} \mathrm{u}_{\mathrm{x}}-\beta\left(\left.\mathbf{v}_{\mathrm{y}}\right|_{\mathbf{r}=(\mathrm{X}, \mathrm{Y})}-\mathrm{u}_{\mathrm{y}}\right)-\partial \mathrm{h} /\left.\partial \mathrm{y}\right|_{\mathbf{r}=(\mathrm{X}, \mathrm{Y})} .
\end{gathered}
$$

It is convenient to present these equations in a dimensionless form. As characteristic length of a problem we will accept the size of a trunk of a vortex $r_{0}$, for characteristic time and speed $-1 / \Omega_{0}$ and $\Omega_{0} \mathrm{r}_{0}$ respectively. Then the equations (18), (19) will become

$$
\begin{gathered}
\mathrm{du}_{\mathrm{x}} / \mathrm{dt}=2 \mathrm{u}_{\mathrm{y}}+\gamma\left(\left.\mathrm{v}_{\mathrm{x}}\right|_{\mathrm{r}=(\mathrm{X}, \mathrm{Y})}-\mathrm{u}_{\mathrm{x}}\right)-\partial \mathrm{h} /\left.\partial \mathrm{x}\right|_{\mathrm{r}=(\mathrm{X}, \mathrm{Y}),} \\
\mathrm{du}_{\mathrm{y}} / \mathrm{dt}=3 \mathrm{y}-2 \mathrm{u}_{\mathrm{x}}+\gamma\left(\left.\mathrm{v}_{\mathrm{y}}\right|_{\mathrm{r}=(\mathrm{X}, \mathrm{Y})}-\mathrm{u}_{\mathrm{y}}\right)-\partial \mathrm{h} /\left.\partial \mathrm{y}\right|_{\mathrm{r}=(\mathrm{X}, \mathrm{Y})},
\end{gathered}
$$

where $\gamma$-dimensionless parameter

$$
\gamma=\beta / \Omega_{0}=18 \rho v / \rho^{*} D^{2} \Omega_{0} .
$$

Let's consider at first the dynamics of particles in the vortex trunk $\left(\mathrm{r}^{2} / \mathrm{r}_{0}^{2}<1\right)$ where the profile of rotation of a vortex has uniform character. In a dimensionless form

$$
\mathrm{v}_{\mathrm{x}}=-\mathrm{Ax}-\omega \mathrm{y}+\mathrm{O}\left(\mathrm{r}^{2} / \mathrm{r}_{0}^{2}\right), \mathrm{v}_{\mathrm{y}}=-\mathrm{Ay}+\omega \mathrm{x}+\mathrm{O}\left(\mathrm{r}^{2} / \mathrm{r}_{0}{ }^{2}\right) .
$$

where A and $\omega$ are measured in units $\Omega_{0}$. From (2) taking into account (13) it is found

$$
\begin{aligned}
& \partial \mathrm{h} / \partial \mathrm{x}=-\left(\mathrm{A}^{2}-\omega^{2}-2 \omega\right) \mathrm{x}-2 \mathrm{~A}(\omega+1) \mathrm{y} \\
& \partial \mathrm{h} / \partial \mathrm{y}=2 \mathrm{~A}(\omega+1) \mathrm{x}-\left(3+\mathrm{A}^{2}-\omega^{2}-2 \omega\right) \mathrm{y}
\end{aligned}
$$

Substituting (23) - (25) in the equations (20) and (21), we will receive the equations of motion of rigid particles in the field of a vortex trunk:

$$
\left\{\begin{array}{l}
\dot{X} \\
\dot{Y} \\
\dot{u}_{x} \\
\dot{u}_{y}
\end{array}\right\}=\left\{\begin{array}{cccc}
0 & 0 & 1 & 0 \\
0 & 0 & 0 & 1 \\
a & b & -\gamma & 2 \\
-b & a & -2 & -\gamma
\end{array}\right\}\left\{\begin{array}{l}
X \\
Y \\
u_{x} \\
u_{y}
\end{array}\right\},
$$

where

$$
a=\mathrm{A}(\mathrm{A}-\gamma)-(\omega+1)^{2}+1 ; \mathrm{b}=2 \mathrm{~A}(\omega+1)-\gamma \omega .
$$

From these equations it is visible that equilibrium position of rigid particles in a vortex trunk is its centre $\mathrm{X}=\mathrm{Y}=0$, where $\mathrm{u}_{\mathrm{x}}=\mathrm{u}_{\mathrm{y}}=0$ and $\dot{u}_{x}=\dot{u}_{y}=0$. Particles in considered area gradually come nearer to the centre of the vortex by helicoidal trajectories.

For establishing the stability of this position of balance, it is necessary to require real parts of eigenvalues of a matrix (26) to be zero or negative.

The matrix of factors (26) has complex eigenvalues of a kind

$$
\Lambda_{1,2,3,4}=-\gamma / 2 \mp \mathfrak{l} \pm \sqrt{ }\left[a-1+\gamma^{2} / 4 \pm \underline{I}(b-\gamma)\right],
$$

After allocation of real part of (28), for stability we receive a condition

$$
(\mathrm{b}-\gamma)^{2}+\gamma^{2}(a-1) \leq 0,
$$

at which centrifugal force is always less than the sum of drag and Coriolis forces, and the resultant force operating on a rigid particle, is directed to the vortex centre.

From (29) the necessary condition of stability follows: $a \leq 1$, which taking into account $(27)$, gives $(\omega+1)^{2}>\mathrm{A}(\mathrm{A}-\gamma)$ that is satisfied for any positive values of $A$ and $\gamma$. The condition (29) with the account (27) leads to stability criterion

$$
\gamma>\mathrm{A}
$$

that taking into account (16), in a dimensional form, for viscosity $v$ gives

$$
v>\rho^{*} \mathrm{AD}^{2} / 18 \rho .
$$

Consider now the question whether there exists an anticyclonic orbit in the volume of Burgers vortex (1) on which the sum of Coriolis and drag forces is counterbalanced by centrifugal force, i.e. in local cylindrical system of co-ordinates

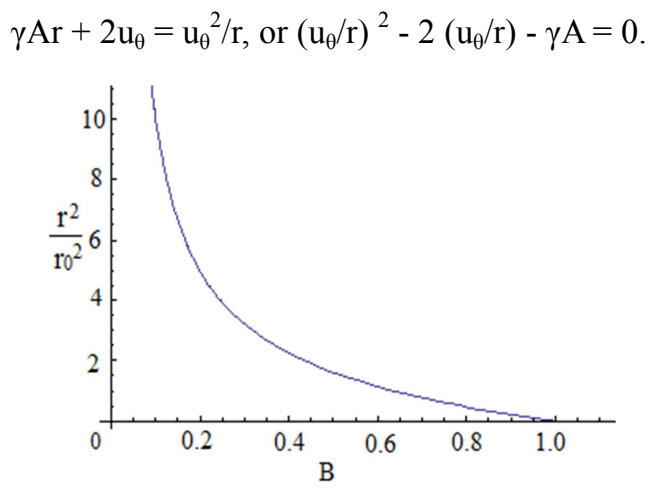

Fig. 4. On the solution of transcendental equation (32). 
It represents the transcendental equation which in a dimensional form looks like

$$
\left[1-\exp \left(-r^{2} / r_{0}^{2}\right)\right] r_{0}^{2} / r^{2}=B,
$$

where

$$
\mathrm{B} \equiv\left[1+\sqrt{ }\left(1+\beta \mathrm{A} / \Omega_{0}{ }^{2}\right)\right] \Omega_{0} / \omega .
$$

The equation (32) has real solutions for orbit radius only at $\mathrm{B} \leq 1$ (fig. 4), and $\mathrm{r}=0$ at $\mathrm{B}=1$. From expression $\mathrm{B}$ it is visible that $\mathrm{B}>2 \Omega_{0} / \omega$. This condition leads to the result $\mathrm{B}>1$. Hence, unique position of balance for rigid particles in a Burgers vortex is its centre where all particles captured by a vortex will gather during the characteristic time

$$
\tau \sim \omega \mathrm{r}_{\mathrm{eff}} / \mathrm{A} \sqrt{ } \beta v
$$

The mass of the rigid particles captured by a vortex is an order

$$
\mathrm{M}_{\mathrm{p}} \approx \pi \mathrm{r}_{\mathrm{eff}}^{2} \Sigma^{*},
$$

which forms a planetesimal.

\section{Discussion}

Let's estimate an order of magnitudes $\tau$ and $\mathrm{M}_{\mathrm{p}}$ for a model of a disk of radius $30 \mathrm{AU}$ and mass $0.5 \mathrm{M}_{\odot}$ round a star of solar mass: $\mathrm{M} \approx \mathrm{M}_{\odot}$, also we will place the local frame of reference at distance $\mathrm{R}_{0}=20 \mathrm{AU}$. This gives estimations $\Omega_{0} \approx 8 \cdot 10^{-9} \mathrm{~s}^{-1}$ and $\Sigma \sim 1600 \mathrm{~g} / \mathrm{cm}^{2}$.

For a typical protoplanetary disk at distance $\mathrm{R}_{0}=20 \mathrm{AU}$. The vertical scale height is of order $\mathrm{H} \approx 10^{8} \mathrm{~km}$. For sound speed, then, we receive an estimation $\mathrm{c}_{\mathrm{s}} \approx \mathrm{H} \Omega_{0} \approx 0.8 \mathrm{~km} / \mathrm{s}$.

Choosing different values for vortex parametres, it is possible to receive different results. For example, accepting the maximum speed of rotation of a vortex $0.01 \mathrm{~km} / \mathrm{s}$ at distance $\mathrm{r}_{0} \approx 10^{7} \mathrm{~km}$ from its center, and speed of a converging stream $\mathrm{v}_{\mathrm{r}}=\mathrm{A} \cdot \mathrm{r}_{0} \approx 0.005 \mathrm{~km} / \mathrm{s}$, we will receive

$$
\omega \approx 10^{-9} \mathrm{~s}^{-1}, \mathrm{~A} \approx 5 \cdot 10^{-10} \mathrm{~s}^{-1} .
$$

The condition of stability of position of rigid particles in the center of a Burgers vortex for viscosity (31) is carried out with a large supply for protoplanetary disks. The matter is that the molecular viscosity of gas, estimated by the formula $v \sim \lambda \mathrm{c}_{\mathrm{s}}$, in which $\lambda$ is the mean free path of molecules, $c_{s}$ is the speed of a sound, does not play an appreciable role in processes of a protoplanetary disk. Really, the mean free path is defined as $\lambda$ $\sim 1 / \mathrm{n} \sigma$, where $\mathrm{n}$ is the concentration of molecules of gas, $\sigma$ is the section of their interaction. Concentration of molecules is equal in the central plane of a disk to $\mathrm{n} \sim \Sigma /\left(2 \mathrm{~m}_{\mathrm{H}} \mathrm{H}\right) \approx 10^{14}$ $\mathrm{cm}^{-3}$. Taking interaction section close to the area of a molecule of hydrogen $\left(\sigma \sim 10^{-16} \mathrm{~cm}^{2}\right)$, we will receive: $\lambda \sim 20 \mathrm{~cm}, \nu \sim$ $10^{6} \mathrm{~cm}^{2} / \mathrm{s}$. Characteristic time of evolution of the disk is of the order of $\tau=R^{2} / \mathrm{v}$, that makes $\sim 10^{13} \mathrm{yrs}$, which is $10^{6}$ times more than the observable time of disk evolution.

For this reason, the ' $\alpha$-disk' [19] model is used to describe accretion disks, in which turbulent viscosity is represented by the expression $v \sim \alpha \mathrm{c}_{\mathrm{s}} \mathrm{H} \approx \alpha \mathrm{H}^{2} \Omega_{0}$, the dimensionless parameter $\alpha$ is considered as constant value of an order $\alpha$ $\sim 10^{-2}$. The scale of viscous length thus makes $\mathrm{L}_{v} \approx 10^{6} \mathrm{~km}$, so Burgers vortex of big sizes cannot be destroyed by viscosity. Keplerian shear length makes $\mathrm{L}_{\text {shear }} \approx 6 \cdot 10^{9} \mathrm{~km}$. Hence, vortices with the sizes $\mathrm{r}_{\text {eff }}<\mathrm{L}_{\text {shear }}$ can have circular form.

Taking into account that $\rho^{*} / \rho \approx 10^{10}$ in a midplane of a disk, using in (33) also the average value for viscosity from stability condition (31), we will receive the following estimations:

$$
\mathrm{M}_{\mathrm{p}} \approx 10^{28} \mathrm{~g} ; \tau \sim 3 \cdot 10^{6}(\mathrm{~m} / \mathrm{D}) \mathrm{yrs} .
$$

So, during an order of $\sim 10^{6} \mathrm{yrs}$, for meter-sized rigid particles, in the vortex trunk the mass amount comparable with mass of Venus accumulates.

\section{Conclusion}

From stated above it follows that the formation of planetesimals by means of an anticyclonic Burgers vortex is effective enough. Operating on rigid particle in such a vortex force of Coriolis, Stokes drag force and pressure gradient force are directed to its center. There is not any circular orbit in centrifugal balance in Burgers vortex in local approach. Only stable position for rigid particle is the vortex center.

Our estimations for a typical protoplanetary disc show that the two-dimensional anticyclonic Burgers vortex with homogeneously rotating kernel and a converging radial stream of substance can effectively accumulate in its nuclear area metersized rigid particles of total mass $\sim 10^{28} \mathrm{~g}$ (or more) for characteristic time $\sim 10^{6} \mathrm{yr}$.

\section{Acknowledgment}

I am grateful to reviewer for his/her helpful suggestions

\section{References}

[1] Kevin Heng, Scott J. Kenyon (arXiv:1005.1660v3 [astro-ph. EP] 17 Lul 2010).

[2] Youdin, A. N., European Astronomical Society (EAS) Publication Series, 41, 187, 2010.

[3] Armitage, P. J., arXiv: astro-ph/0701485v2, 2007.

[4] Armitage, P. J., Astrophysics of Planet Formation, Cambridge University Press, UK, 2010.

[5] Blum, J., Wurm, G., ARA\&A, 46, 21, 2008.

[6] Zsom, A., Ormel, C. W., Guttler, C., Blum, J., Dullemond, C. P., arXiv: 1001.0488v1, 2010.

[7] Wilner, D. J., D’Alessio, P., Calvet, N., Claussen, M. J., Hartmann, L., ApJ, 626, L109, 2005.

[8] Adachi, I., Hayashi, C., Nakazawa, K., Prog. Theor. Phys., 56, 1756, 1976

[9] Weidenschilling, S. J., MNRAS, 180, 57, 1977. 
[10] Paterson, H. L., Feng, M., Waite, A. M., Gomis, D., Beckley, L. E., Holliday, D., Thompson, P. A., Journal of Geophysical Research, 113, C7, C07049, 2008.

[11] Carnevale, G. F., McWilliams, J. C., Pomeau, Y., Weiss, J. B., Young, W. R., Phys. Rev. Lett., 66, 2735, 1991.

[12] Weiss, J. B., McWilliams, J. C., Phys. Fluids A, 5, 3, 1993.

[13] Tabeling, P., Physics Reports, 362, 1, 2002.

[14] L\&mro, B. J., Lasheras, J. C., J. Fluid Mech. 235, 179, 1992.

[15] Inaba, S., Barge, P., Daniel, E., Guillard, H., A\&A, 431, 365, 2005

[16] Inaba, S., Barge, P., Ap. J., 649, 415, 2006
[17] Abrahamyan, M. G., Astrophysics, 51, 201, 2008,

[18] Abrahamyan, M. G., Matveenko, L. I., Astrophysics, 55, 443, 2012.

[19] Shakura, N. I., Sunyaev, R. A., A\&A, 24, 337, 1973.

[20] Pringle, J. E., ARA\&A, 19, 137, 1981.

[21] Chiang, E. I., Goldreich, P., ApJ, 490, 368, 1997.

[22] Godon, P., Livio, M., ApJ, 537, 396, 2000.

[23] Abrahamyan, M. G., Astrophysics, 59, 309, 2016.

[24] Chandrasekhar, S., Ellipsoidal Figures of Equilibria, Yale University Press, 1969. 